\title{
Non-linear dynamics of a viscoelastic film subjected to a spatially periodic electric field
}

\author{
George Karapetsas*, Vasilis Bontozoglou \\ Department of Mechanical Engineering, University of Thessaly, Volos 38334, Greece
}

\section{A R T I C L E I N F O}

Article history:

Received 31 July 2014

Received in revised form 16 December 2014

Accepted 31 December 2014

Available online 10 January 2015

\section{Keywords:}

Electrohydrodynamic instability

Viscoelastic fluid

Numerical simulation

Non-linear dynamics

\begin{abstract}
A B S T R A C T
We investigate the non-linear dynamics of the electrohydrodynamic instability of a viscoelastic polymeric film under a patterned mask. We develop a computational model and carry out 2D numerical simulations fully accounting for the flow and electric field in both phases. We perform a thorough parametric study and investigate the influence of the various rheological parameters, the applied voltage and the period of the protrusions of the mask in order to define the fabrication limits of this process in the case of patterned electrodes. Our results indicate that the effect of elasticity is destabilizing, in agreement with earlier studies in the literature based on linear stability analysis for homogeneous electric fields. However, the significance of the normal and shear polymeric stress components is found to change drastically as deformation advances, rendering inappropriate the lubrication approximation that neglects normal stresses. We also find that for low values of the $C a$ number a metastable state arises with finite interfacial deformation, the amplitude of which compares favourably with experimental observations in contrast with earlier predictions using linear theory. Though the critical voltage for this metastable state appears to be unaffected by the elasticity of the material, viscoelasticity affects the fabrication limit on the period of the protrusions of the top electrode.
\end{abstract}

(C) 2015 Elsevier B.V. All rights reserved.

\section{Introduction}

The interaction of an externally applied electric field with a liquid can give rise to interesting flow instabilities and pattern formation [1]. The work of Russel and co-workers [2-6] has demonstrated that the application of an electric field to an initially flat polymer-air or polymer-polymer interface results in an electrohydrodynamic (EHD) instability leading to the formation of columnar structures. These instabilities can be used in order to form wellcontrolled patterns at the microscale and nanoscale with many practical engineering applications.

The electrically-induced flow of thin liquid films has attracted the interest of many theoretical studies. More specifically, Schaffer et al. [3] used the lubrication approximation to determine the dependence of the fastest growing linear mode on system parameters for a polymer-air interface. Lin et al. [4] conducted experimental as well as theoretical work to study the dependence of pattern wavelength on the viscosity ratio in two-layer polymeric systems. Their modelling study predicts the wavelength to be independent of the viscosity ratio for perfect dielectric fluids. Pease and

\footnotetext{
* Corresponding author.

E-mail addresses: gkarapetsas@gmail.com (G. Karapetsas), bont@mie.uth.gr (V Bontozoglou).
}

Russel [7] considered the stability of the interface between a leaky dielectric liquid and air and showed that the presence of conductivity exerts a destabilizing influence leading to patterns of smaller wavelength and much larger growth rates. Shankar and Sharma [8] also conducted a linear stability analysis using lubrication theory and their results indicate that, in contrast to the perfect dielectric case, for leaky dielectrics, increasing the viscosity ratio has a profound influence on the pattern wavelength.

More recently, Heier et al. [9] were interested in systems with heterogeneous electric fields and showed through experiments that it is possible to achieve a steady state with finite interfacial deformation when Maxwell stresses in the fluids and surface tension are balanced. They also developed a linear model and were able to derive an expression for the critical voltage beyond which the amplitude grows exponentially in qualitative agreement with their experiments. However, it should be noted that according to Heier et al. [9] linear theory severely underestimates the amplitude of the steady finite deformations in comparison with experimental observations.

The nonlinear evolution of two leaky dielectric layers in a homogeneous electric field was examined by Craster and Matar [10] showing that initially small perturbations grow under the action of the destabilizing electrical forces and eventually their amplitude saturates in the non-linear regime to give rise to spatially periodic 
patterns. Two-dimensional numerical simulations using the lubrication theory helped in elucidating the interfacial evolution, the role of the initial thickness ratio and the effect of patterned "masks" on the observed three-dimensional patterns [11-14]. Several studies have also been devoted in the investigation of the stability and dynamics of bilayers under air or another viscous liquid [15-19]. Finally, the effect of AC fields has been taken into account through linear stability analysis and non-linear simulations by Roberts and Kumar [20] and Gambhire and Thaokar [21].

As discussed above, the surface instability of a Newtonian fluid under the effect of electric field has been studied extensively by several researchers and it is now well understood. The dynamics of fluids with complex rheology, however, has received much less attention in the literature. The first attempt to take into account the polymer viscoelasticity in electrically-induced flows was made by $\mathrm{Wu}$ and $\mathrm{Chou} \mathrm{[22].} \mathrm{These} \mathrm{researchers} \mathrm{used} \mathrm{the} \mathrm{lubrication} \mathrm{theory}$ and performed a linear stability analysis of a initially static thin polymer film underneath a flat electrode using the Oldroyd-B constitutive equation for the elastic stresses. Their results have shown that the polymer elasticity destabilizes the system and when the Deborah number is large enough, a resonant phenomenon appears as a result of the interaction between the two destabilizing mechanisms (the electrostatic force and the polymer elasticity). Later on, Tomar et al. [23] used a linear constitutive equation for the stresses (Jeffreys model) and presented a linear stability analysis taking also into account the effect of inertia. Interestingly, they found that in the presence of a small amount of inertia the wavelength of the fastest growing mode (i.e. the dominant lengthscale of the instability) is independent of the rheological properties such as relaxation time and solvent viscosity whereas the growth rate is affected significantly. Their findings were confirmed recently by Espin et al. [24] using an asymptotic expansion. The latter authors also examined the viscoelastic effects under the influence of AC fields and found that the impact is largest when the relaxation time and oscillation time scale are comparable. In the case of AC fields, it is shown that the wavelength is also affected contrary to the predictions of linear theory for the case of DC fields [23]. The rheological characteristics of the fluid were also shown to play a role in the case of trilayers, indicating that its effect on the evolution of two coupled interfaces is more involved than a purely kinetic role [25]. It should be noted here that the aforementioned studies for viscoelastic fluids considered homogeneous electric fields (flat electrodes) and the linear stability analysis was performed around a quiescent base state. However, in the case of a patterned mask the field becomes heterogeneous and growth generates a time-dependent base state for which linear or weakly non-linear stability analysis is difficult necessitating the use of time-dependent simulations.

As was noted above, most of the research studies in the literature employ linear theory, which is valid only for small disturbances. One crucial issue, however, is not only to predict the band of unstable wavenumbers in the linear regime but also to determine accurately the behaviour of the system in the non-linear regime. For the latter, the majority of the research groups make use of the lubrication theory in order to interpret experimental results. Pease and Russel $[26,27]$ argued, however, that in many cases the experiments were carried out for conditions under which the lubrication approximation is not strictly valid. They compared the predictions of a generalised model with those of lubrication theory against experimental results and found a better agreement with the former. Very recently, a detailed comparison was also presented by Gambhire and Thaokar [21] for both DC and AC fields, which indicated large deviations for the predicted wavelength. Moreover, in the case of viscoelastic fluids, the deficiencies of the lubrication approximation are expected to be enhanced due to the significant underestimation of normal stresses and to the fact that non-linear viscoelastic effects are not taken into account.
Examples of fully non-linear simulations without making use of the lubrication approximation are the works of [28-31] who studied primarily cases involving heterogeneous electric fields. Yang et al. [30], motivated by the work of Heier et al. [9], considered a sinusoidally patterned top electrode and performed non-linear simulations using a boundary/finite element method to determine the critical parameters for instability of the liquid film. Their results indicate that linear analysis can significantly over-predict the critical voltage for instability. Li et al. [31] were also interested in heterogeneous electric fields and investigated the effect of various geometric features of the patterned electrode to determine the fabrication limits of this process using a diffuse interface method.

The scope of this work is to investigate the non-linear dynamics of a viscoelastic material under the influence of an heterogeneous electric field taking fully into account the viscoelastic effects. We avoid making any assumptions, such as using lubrication approximation, in order to describe the flow dynamics as accurately as possible. We perform two-dimensional transient numerical simulations, using the finite element method combined with an elliptic grid generation scheme for the determination of the unknown position of the interface. The viscoelasticity of the polymeric film is taken into account using the affine Phan-Thien Tanner model. We perform an extensive parametric analysis to determine the effects of the various geometric and rheological parameters on the evolution of the interface and on the fabrication limits of this process. Our results indicate that the elasticity of the material does not affect the critical voltage for instability but affects the fabrication limit on the period of the top electrode protrusions. We also discuss about the validity of lubrication theory in the case of viscoelastic materials.

The remainder of the paper is organized as follows. In Section 2, we describe the system of governing equations and outline the numerical method used for its numerical solution. The results are presented and discussed in Section 3. Finally, the concluding remarks are given in Section 4.

\section{Problem formulation}

We consider the dynamics of two perfect dielectric fluids sandwiched between two rigid, and impermeable electrodes. The electrodes can be either flat or periodically patterned as shown in Fig. $1 ; w$ and $p$ denote the width and the height of the protrusions, respectively, and $s$ denotes the spacing between the protrusions. The bottom fluid is considered to be a polymeric viscoelastic film surrounded by a Newtonian liquid, with initial thickness, $d$. Both fluids, which are initially stationary, are taken to be incompressible with the lower (upper) fluid having a density $\rho_{1}\left(\rho_{2}\right)$, dielectric constants $\epsilon_{1}\left(\epsilon_{2}\right)$; these properties are assumed to be constant. The viscosity of the upper fluid is also constant and denoted by $\mu_{2}$. The viscoelastic fluid has a zero-shear viscosity $\mu_{1}=\mu_{s}+\mu_{p}$, where $\mu_{s}$ and $\mu_{p}$ are the viscosities of the solvent and the polymer,

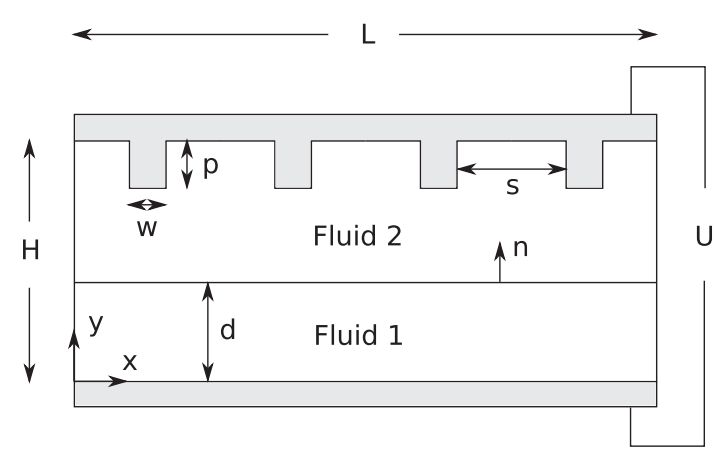

Fig. 1. Schematic of the flow geometry. 
respectively, and relaxation time $\lambda$. The surface tension of the liquid-liquid interface, $\sigma$, is assumed to be constant. The top and bottom electrodes are maintained at constant potentials $\phi_{2}(y=H(x))=U$ and $\phi_{1}(y=0)=0$, respectively.

We scale all lengths with the maximum distance between the top and bottom electrodes, $H_{m}$, velocities with $V^{*}=\epsilon_{o} U^{2} /\left(\mu_{1} H_{m}\right)$, pressure and stresses with $\mu_{1} V^{*} / H_{m}$ and electric potential with the potential difference $U$. Substituting this scaling into the governing equations and boundary conditions, the dimensionless groups that emerge are the Reynolds number, $R e=\rho_{1} V^{*} H_{m} / \mu_{1}$, the Stokes number, $S t=\rho_{1} g H_{m}^{2} /\left(\mu_{1} V^{*}\right)$, the capillary number, $C a=\mu_{1} V^{*} / \sigma$, the Weissenberg number, $W i=\lambda V^{*} / H_{m}$, which is a measure of the elasticity of the polymeric liquid, the density ratio, $D_{i}=\rho_{i} / \rho_{1}$, the viscosity ratio, $M_{i}=\mu_{i} / \mu_{1}$, and the solvent viscosity ratio of the polymer liquid, $\beta=\mu_{s} / \mu_{1}$.

Inserting the previously defined characteristic quantities into the momentum and mass conservation equations, we obtain:

$D_{i} \operatorname{Re}\left(\frac{\partial \mathbf{v}_{i}}{\partial t}+\mathbf{v}_{i} \cdot \nabla \mathbf{v}_{i}\right)-\nabla \cdot T_{i}+$ Ste $_{z}=0$

$\nabla \cdot \mathbf{v}_{i}=0$

where $\nabla$ denotes the gradient operator, subscript $i$ indicates the corresponding fluid, $\mathbf{v}_{i}$ is the velocity vector and $T_{i}$ is the total stress tensor given by

$T_{i}=-P_{i} I+\tau_{i}+m_{i}$

Here $P_{i}$ denotes the pressure, $I$ is the identity tensor and $\tau_{i}$ the extra stress tensor

$\tau_{1}=\tau_{p, 1}+2 \beta M_{1} \gamma_{1}$

$\tau_{2}=2 M_{2} \gamma_{2}$

Note that for the viscoelastic material the extra stress tensor is split into a purely viscous part, $2 \beta M_{1} \gamma_{1}$, and a polymeric contribution, $\tau_{p, 1} \cdot \gamma_{i}$ denotes the rate of strain tensor

$\gamma_{i}=\frac{1}{2}\left(\nabla \mathbf{v}_{i}+\nabla \mathbf{v}_{i}^{T}\right)$.

The Maxwell stress tensor, denoted by $m_{i}$, describes the interaction of fluid $i$ with the electric field, $\mathbf{E}_{i}$, and is defined as

$m_{i}=\epsilon_{i} \mathbf{E}_{i} \mathbf{E}_{i}-\frac{1}{2} \epsilon_{i} \mathbf{E}_{i} \cdot \mathbf{E}_{i} I$.

Note that due to the absence of free charge from the bulk of the fluid and since $\epsilon_{1}$ and $\epsilon_{2}$ are spatially independent, $\nabla \cdot m_{i}=0$. Therefore it becomes evident that Maxwell stresses will not have any contribution in Eq. (1) but nevertheless enter the problem through the interfacial boundary conditions. The Maxwell stresses depend on the local intensity of the electric field. Under the electrostatic approximation and for an electrically neutral fluid, Maxwell's equations reduce to the following set of equations

$\nabla \cdot\left(\epsilon_{i} \mathbf{E}_{i}\right)=0$

$\nabla \times \mathbf{E}_{i}=0$.

Since the electrical field is irrotational, we can define a potential function $\phi_{i}$ such that

$\mathbf{E}_{i}=-\nabla \phi_{i}$

By combining Eqs. (8) and (10) we get the following equation

$\nabla \cdot\left(\epsilon_{i} \nabla \phi_{i}\right)=0$.

To complete the description, a constitutive equation that describes the rheology of the viscoelastic material is required in order to determine the polymeric part of the extra stress tensor. As such, we use the following differential model that has been proposed by [32]
$Y\left(\tau_{p, 1}\right) \tau_{p, 1}+W i \hat{\tau}_{p, 1}=2(1-\beta) M_{1} \gamma_{1}$.

The symbol ${ }^{\wedge}$ over the viscoelastic stress denotes the GordonSchowalter derivative defined as

$\widehat{X}=\frac{\partial X}{\partial t}+\mathbf{v}_{1} \cdot \nabla X-\left(\nabla \mathbf{v}_{1}-\xi_{s} \gamma_{1}\right)^{T} \cdot X-X \cdot\left(\nabla \mathbf{v}_{1}-\xi_{s} \gamma_{1}\right)$,

where $X$ is any second order tensor. Two forms of the PTT model are in common use, the linearised form [32], where the function $Y\left(\tau_{p, 1}\right)$ is

$Y\left(\tau_{p, 1}\right)=1+\frac{a_{\text {PTT }} W i}{(1-\beta) M_{1}} \operatorname{tr}\left(\tau_{p, 1}\right)$,

and the exponential form [33]

$Y\left(\tau_{p, 1}\right)=\exp \left[\frac{a_{P T T} W i}{(1-\beta) M_{1}} \operatorname{tr}\left(\tau_{p, 1}\right)\right]$.

In the present study we have used the exponential form of the PTT model. Both versions of the PTT model have two parameters, $\xi_{s}$ and $a_{\text {PTT }}$. The first one is related to the non-affine motion of the polymer chains with respect to the macroscopic motion of the continuum. By setting $\xi_{s}$ equal to zero no such motion or slip is allowed; for the rest of the paper $\xi_{s}$ will be considered to be zero. The GordonSchowalter derivative reduces to the upper convective one and the fluid model is referred to as the affine PTT model. The second parameter, $a_{P T T}$, imposes an upper limit to the elongational viscosity, which increases as this parameter decreases, while it introduces elongational thinning. Moreover $a_{P T T}$ is related to the shear-thinning behaviour of the model. The predictions for the elongational and shear viscosity of this model for various values of $a_{P T T}$ appear in Fig. 9 of [34]. By setting both $a_{P T T}=0$ and $\xi_{S}=0$, the PTT model reduces to the Oldroyd-B model. Retaining the zero values for $a_{P T T}$ and $\xi_{s}$ and additionally setting $\beta=0$, the PTT model reduces to the UCM model.

In order to solve accurately and efficiently the flow inside the viscoelastic material we employ the elastic viscous split stress EVSS-G formulation $[35,36]$. This method consists of splitting the polymeric part of the extra stress tensor into a purely elastic and a viscous part

$\tau_{p, 1}=\Sigma+2(1-\beta) M_{1} \gamma_{1}$.

Moreover, an independent interpolation of the components of the velocity gradient tensor is introduced

$G=\nabla \mathbf{v}_{1}$.

The former splitting ensures the elliptic nature of the momentum equations even in the absence of a solvent $(\beta=0)$, while the latter substitution makes the approximations in the constitutive equation of the elastic stress and the velocity gradient compatible to each other. This scheme has been used with success in the past $[34,37,38]$ permitting the calculations up to very high Weissenberg numbers.

\subsection{Boundary conditions}

Solution of the above set of equations is determined subject to the following boundary conditions.

On the solid walls we apply the usual no-slip and no-penetration boundary conditions while on the two edges of the physical domain $(x=0, L)$ we apply periodic conditions.

Along the liquid-liquid interface the velocity is continuous

$\mathbf{v}_{1}=\mathbf{v}_{2}$

and the flow field satisfies the local interfacial force balance between the stresses in both liquids

$\mathbf{n} \cdot T_{1}=\mathbf{n} \cdot T_{2}+\frac{2 \mathcal{H} \mathbf{n}}{C a}$, 
where $\mathbf{n}$ denotes the unit normal pointing towards the upper liquid, $2 \mathcal{H}$ is the mean curvature of the interface

$2 \mathcal{H}=-\nabla_{s} \cdot \mathbf{n}$,

and $\nabla_{s}$ is the surface gradient operator, defined as

$\nabla_{s}=(I-\mathbf{n n}) \nabla$.

In addition, along the moving interface we impose the kinematic boundary condition,

$\frac{\partial f}{\partial t}+\mathbf{v}_{i} \cdot \nabla f=0$

where $f$ describes the position of the interface. A balance of the normal and tangential component of the electric field gives the continuity of the potentials and the electric displacement across the interface

$\phi_{1}=\phi_{2}$,

$\mathbf{n} \cdot\left(\epsilon_{1} \nabla \phi_{1}\right)=\mathbf{n} \cdot\left(\epsilon_{2} \nabla \phi_{2}\right)$.

Finally to complete our model we have to set a datum pressure and as such, we impose a zero value to the pressure at a node of the top electrode, $P(x=0, y=1)=0$. Initially the film is considered to be static and the liquid-air interface is flat; no initial perturbation is applied.

\subsection{Elliptic grid generation}

The above set of equations is combined with an elliptic grid generation scheme capable of following the deformations of the physical domain. This method has been successfully applied in flows that exhibit large deformations in steady state [37] and transient calculations [39-41]. The grid generation scheme consists of a system of quasi-elliptic partial differential equations, capable of generating a boundary fitted discretization of the deforming domain occupied by the liquid. With this scheme the physical domain $(x, y)$ is mapped onto a computational one $(\eta, \xi)$. A fixed computational mesh is generated in the latter domain while, through the mapping, the corresponding mesh in the physical domain follows its deformations. The mapping is based on the solution of the following system of quasi-elliptic partial differential equations

$\nabla \cdot(\alpha \cdot \nabla \eta)=0$

$\nabla \cdot\left[\left(\delta \sqrt{\frac{x_{\xi}^{2}+y_{\xi}^{2}}{x_{\eta}^{2}+y_{\eta}^{2}}}+(1-\delta)\right) \nabla \xi\right]=0$

where

$\alpha=\left(\begin{array}{ll}1 & 0 \\ 0 & a\end{array}\right), \quad a \geqslant 1$

The subscripts denote differentiation with respect to the indicated variable. The parameter $a$ forces the $\xi$-coordinate lines to be equidistant in the $y$-direction and $\delta$ is a parameter that controls the smoothness of the mapping relative to the degree of orthogonality of the mesh lines. These parameters are adjusted by trial and error; here we set $a=100$ and $\delta=0.1$. For a more detailed description of this method the interested reader may refer to [42-44].

In order to solve the above system of differential equations, appropriate boundary conditions must be imposed. On the fixed boundaries, we impose the equations that define their position, and the remaining degrees of freedom are used for optimally distributing the nodes along these boundaries. Along the moving liquid-liquid interface we simply impose the kinematic equation.

We should note here that special care was taken for the mesh near the interface. In order to resolve adequately the flow, a more refined mesh around this region is needed. To this end, we have introduced a local refinement scheme using the h-method [45]. In order to illustrate the quality of the resulting mesh produced following our method we present in Fig. 2 a typical grid; here we show for clarity the domain $1<x<3$.

In order to solve numerically the governing equations along with the elliptic grid equations, we used the mixed finite element method; this numerical scheme has been used with success in the past [34,37-40]. Detailed information about the weak formulation of all the equations is given in the Appendix A. Finally, the set of algebraic equations is integrated in time with the implicit Euler method introducing an automatic adaptation of the time step for ensuring the convergence of the above iteration scheme and optimizing code performance.

\section{Results}

As shown in Fig. 1 we consider a periodically patterned electrode as a mask to induce the fabrication of similar structures in the liquid. For the purposes of this study we will consider that the width and the height of the protrusions are $w=0.2$ and $p=0.2$, respectively. Since the effect of these geometric characteristics have been discussed in detail for the case of a Newtonian liquid by [31], we will keep these values constant and focus our attention on the remaining parameters of our model. The size of the domain will be considered to be $L=4(s+w)$ unless stated otherwise. For the rest of the paper we will also consider creeping flow conditions and set $R e=10^{-5}$, ignore gravitational effects, $S t=0$, and consider density and viscosity ratios typical of liquidair systems, $D_{2}=0.001, M_{2}=0.001$.

\subsection{Newtonian fluid}

To set the stage for the discussion that follows, it is useful to examine first the case of a Newtonian fluid $(W i=0)$. In Fig. 3 we present contour plots for the velocity field, pressure and electric potential at $t=26.04$ for $C a=30, d=0.3$ and $s=0.8$; for clarity we present here only part of our computational domain $(1 \leqslant x \leqslant 3)$. The liquid film is initially flat and quiescent. Upon the application of voltage the liquid experiences non-uniform electrostatic forces, due to the spatial heterogeneity of the electric field that is created by the top electrode, and liquid is drawn towards the protrusions, destabilizing the liquid-air interface. The flow field appears to be symmetric around the protrusions giving rise to symmetric structures that follow closely the geometrical characteristics of the top electrode. The pressure varies mainly inside the liquid phase (see Fig. 3c) and becomes minimum at the crest, whereas in the gas phase it remains almost constant (approximately equal to the datum pressure) due to the fact that the viscosity of the gas is very small. The contour lines of the electric

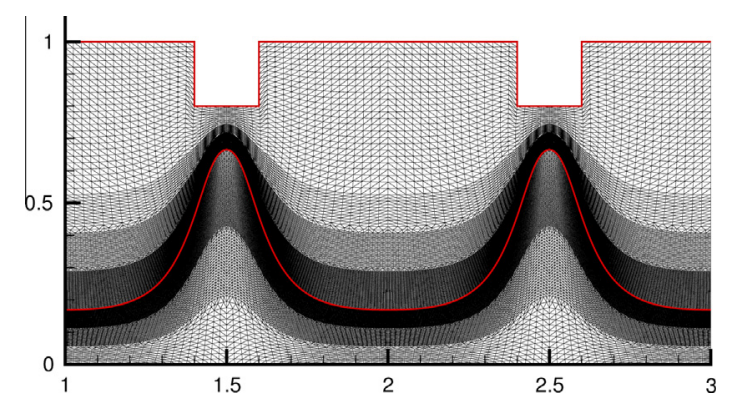

Fig. 2. Typical grid with 3 levels of local refinement adjacent to the liquid-liquid interface for $W i=0, C a=30, d=0.3, s=0.8, p=0.2, w=0.2, L=4$ at time $t=26.04$. 
(a)

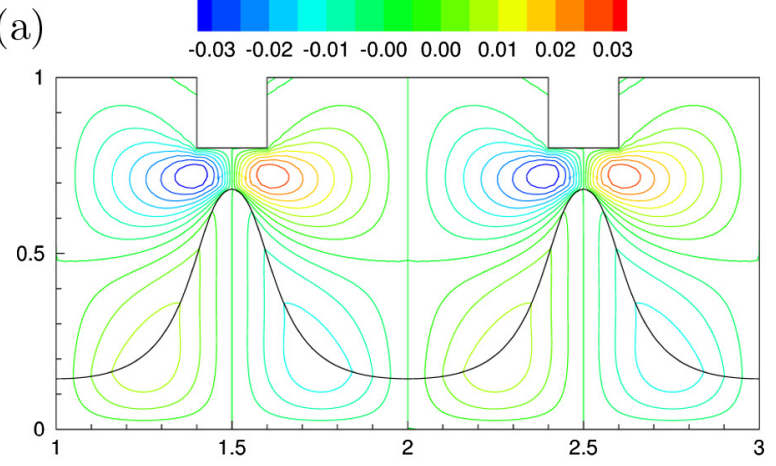

(c)

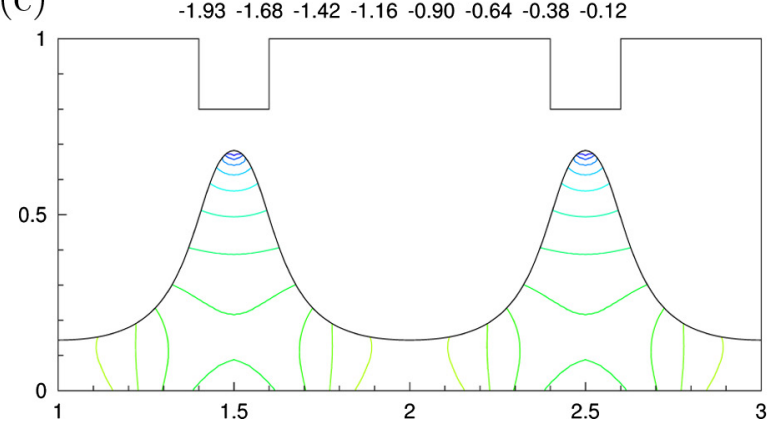

(b)

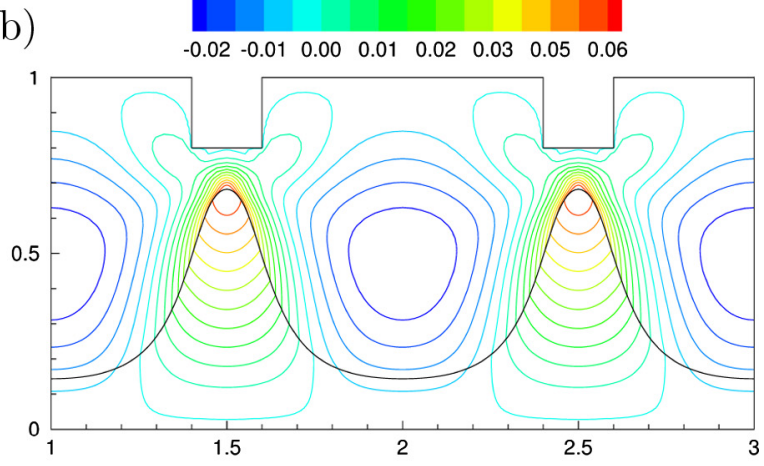

(d)

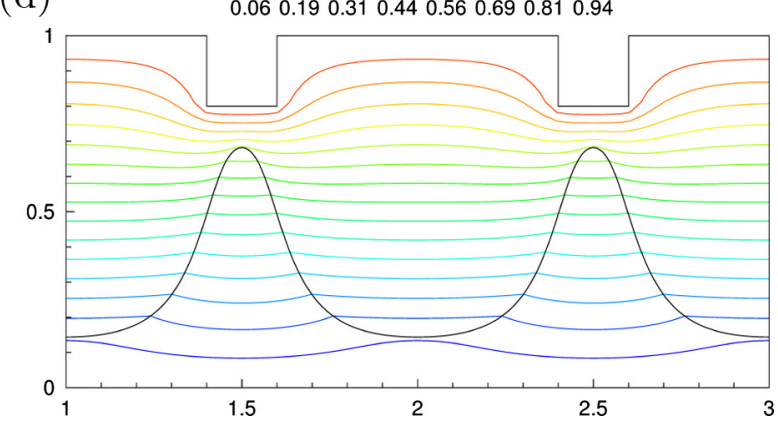

Fig. 3. Contour plots of (a) $v_{x}$, (b) $v_{y}$, (c) $P$ and (d) electric potential at $t=26.04$ for $W i=0, C a=30, \epsilon_{1}=2.5, \epsilon_{2}=1, d=0.3, s=0.8, p=0.2, w=0.2, L=4$.

potential are depicted in Fig. 3d. The deflection of the equipotential lines at the liquid-air interface is due to the difference of dielectric properties of the materials.

For the given set of parameters the height of the interface below the protrusions increases continuously until the liquid comes into contact with the top electrode; the simulation is stopped before we actually reach that point. Heier et al. [9] have shown that by manipulating the combined effect of Maxwell stresses and surface tension it is possible to achieve a steady state interfacial deformation avoiding contact with the top electrode. In order to investigate this possibility, we examine in Fig. 4a the parametric effect of the capillary number, $\mathrm{Ca}$, in the temporal evolution of the maximum amplitude of the interfacial perturbation. Recall that $\mathrm{Ca}$ compares electric to capillary stresses. For the lowest $\mathrm{Ca}$ we confirm that the amplitude initially grows and then saturates, indicating that electric and capillary forces balance exactly. This situation evidently corresponds to a static equilibrium, as there is no driving force for flow. The equilibrium amplitude increases with $\mathrm{Ca}$ as depicted in Fig. 4c, and its maximum values are in the order of $10 \%$ of the electrode spacing.

According to the linear theory developed by Heier et al. [9] the dimensionless amplitude $Z=\zeta_{0} \sqrt{k^{2}\left(1-d+d / \epsilon_{p}\right) / C a}$, where $\zeta_{0}=\left(h_{\max }-h_{\min }\right) / 2$ and $k=2 \pi /(s+w)$ should depend linearly on $\epsilon \tau /\left(1-\tau^{2}\right), \quad$ where $\quad \epsilon=p /\left[2\left(1-d+d / \epsilon_{p}\right)\right] \quad$ and $\tau=\left(1-1 / \epsilon_{p}\right) \sqrt{C a /\left[k^{2}\left(1-d+d / \epsilon_{p}\right)^{3}\right]}$. Heier et al. [9] correlated experimental data of $Z$ by a power-law dependence on $\epsilon \tau /\left(1-\tau^{2}\right)$, and found that the exponent is not equal to one -as expected from their linear theory- but actually is much higher, approximately equal to 4.5 . The results of our non-linear calculations, plotted in terms of the above dimensionless parameters, are shown in Fig. 4b. We observe that the correlation by a power-law is not completely satisfactory, as the slope in log-log coordinates gradually increases. However, an approximate value for the exponent is 3.2, which is significantly higher than the linear prediction and a bit lower than the experimental value.

The long-time results depicted in Fig. 4a indicate that the equilibrium state is not stable but is eventually destroyed and some peaks reach the upper electrode. The process by which the interface disintegrates is shown in Fig. 5 for $\mathrm{Ca}=8$ and involves loss of the spatial periodicity imposed by the electrode spacing. We note however that the time-scale for this process is at low $\mathrm{Ca}$ an order of magnitude higher than the time-scale for growth of the equilibrium amplitude. Thus, the equilibrium state may actually be considered as metastable.

Increasing the $\mathrm{Ca}$ number, the effect of surface tension becomes less significant and the destabilization of the interface takes place sooner. Plotting the time that is needed for $h_{\max }-h_{\min }=0.4$ (a convenient arbitrary criterion) it is possible to evaluate the threshold in the $\mathrm{Ca}$ number beyond which the metastable state is not observed (see Fig. 4b) and we find that for the given set of parameters it is approximately equal to $C a \approx 10$. According to linear theory $[9,30]$ the critical $\mathrm{Ca}$ can be evaluated using the following expression

$C a_{c r}=k^{2} \frac{\left[\epsilon_{p}(1-p / 2-d)+d\right]^{3}}{\epsilon_{p}\left(\epsilon_{p}-1\right)^{2}}$,

where $k$ is the wavenumber of the interfacial perturbation which is assumed to largely follow the pattern of the top electrode. For the given set of parameters and assuming that the dimensionless wavelength of the initial disturbance is approximately equal to $s+w$, we get $C a_{c r}=40.89$. It appears that linear theory significantly over-predicts the critical voltage and this was also found to be true in the non-linear simulations presented by [30].

The loss of interfacial periodicity that is observed in the evolution of the metastable equilibrium appears to be a more general characteristic of the present problem. In support of this argument, 

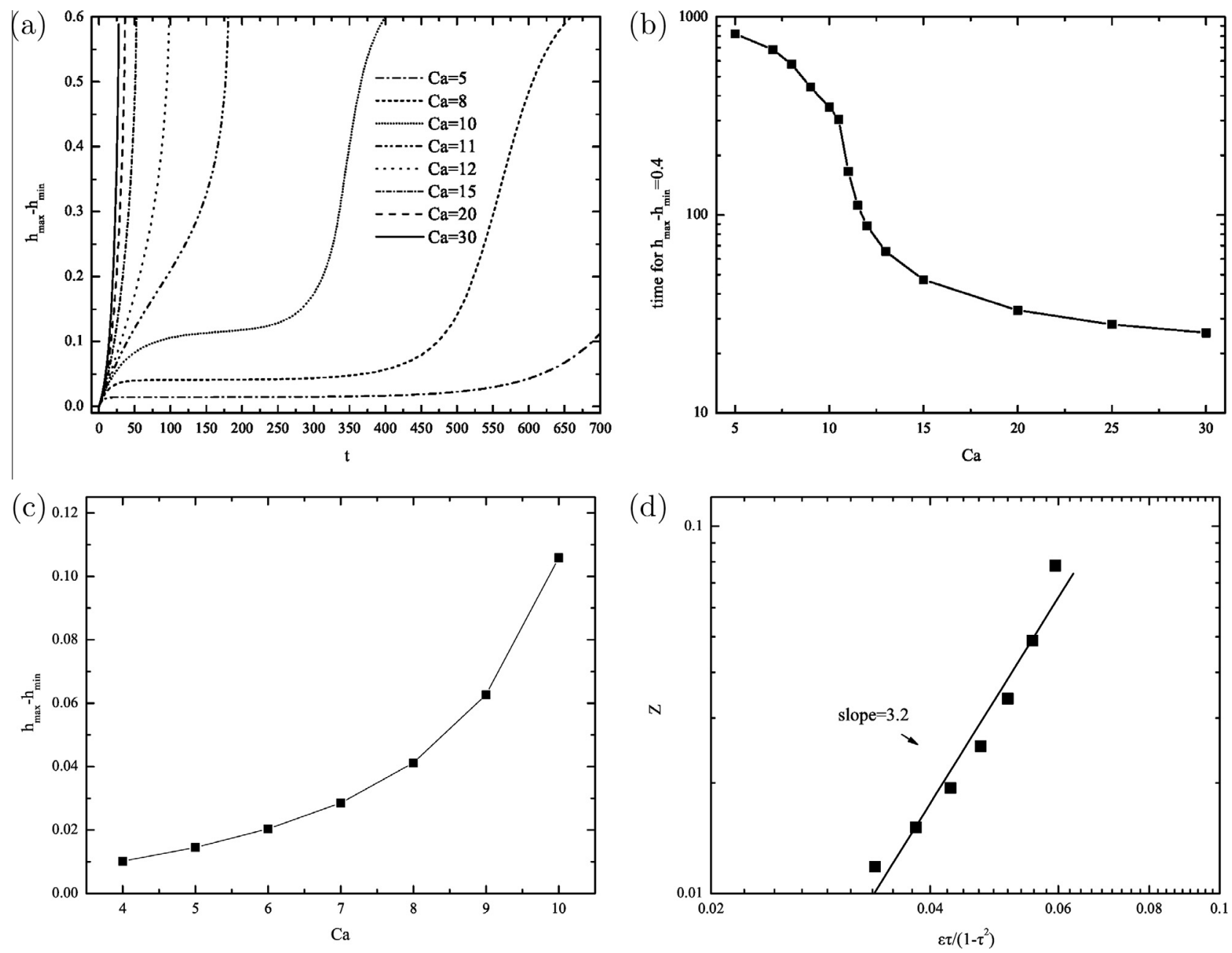

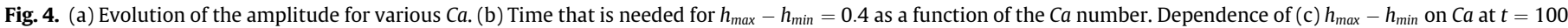
for various $W i$ and (d) the dimensionless amplitude $Z$ on $\epsilon \tau /\left(1-\tau^{2}\right)$. The remaining parameters are $W i=0, \epsilon_{1}=2.5, \epsilon_{2}=1, d=0.3, s=0.8, p=0.2, w=0.2, L=4$.

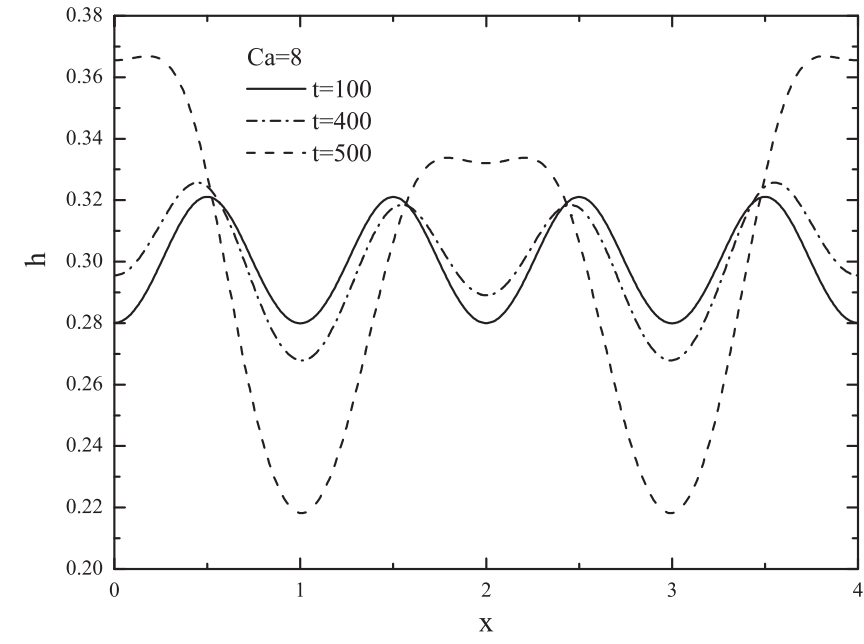

Fig. 5. Profiles of the liquid-air interface for $C a=8$ at $t=100,400,500$. $\mathrm{Wi}=0, \epsilon_{1}=2.5, \epsilon_{2}=1, d=0.3, s=0.8, p=0.2, w=0.2, L=4$.

we present in Fig. 6 the long-time profiles of the liquid-air interface for three different values of the $\mathrm{Ca}$ number. We observe that for values higher than the 'critical' $\mathrm{Ca}$ the deformation of the interface remains periodic, following closely the spatial periodicity
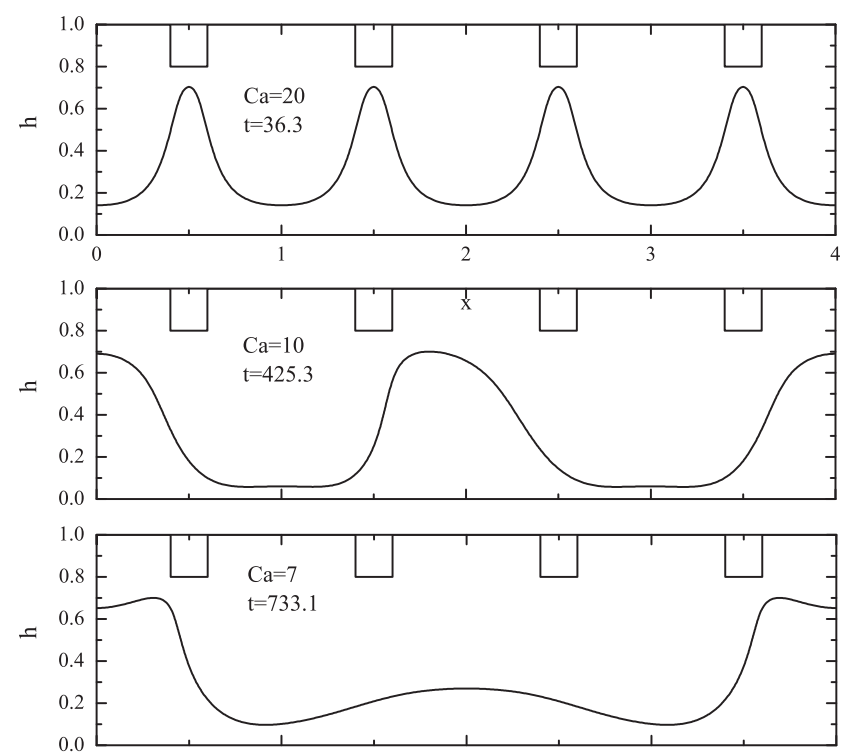

Fig. 6. Long time profiles of the liquid-air interface for various $\mathrm{Ca}$ and for $W i=0, \epsilon_{1}=2.5, \epsilon_{2}=1, d=0.3, s=0.8, p=0.2, w=0.2, L=4$. 
imposed by the top electrode, during the entire evolution toward formation of columnar structures. On the other hand, for low $\mathrm{Ca}$ the spatial periodicity of the interface is eventually destroyed, despite the initial periodicity of the metastable state. The evolution is always a coarsening process similar to the one described in [46]. More specifically, some pillars expand at the expense of shrinkage of their neighbours and this is essentially an Ostwald ripening phenomenon.

The mechanism responsible for the ripening process can be rationalized as follows. Initially, the wavelength of the interface perturbation is imposed by the pattern of the electrode which induces non-uniform Maxwell stresses to the interface. Note that for the parameters that have been used in this study the wavelength is 2-5 times smaller than the wavelength of the fastest growing mode which is predicted by linear theory for flat electrodes. Even though this represents a barrier which is set by the effect of surface tension it can be overcome due the effect of the heterogeneous electric field. For cases that growth is sufficiently fast, the mode which is imposed by the patterned electrode will dominate and the pattern of the electrode will be replicated in the film retaining its periodic structure. On the other hand, if growth is relatively slow or the system reaches equilibrium the mode of the natural wavelength, which is dominated by the effect of surface tension, is given the time to grow and eventually dominate the flow.

One question that arises is whether these non-periodic solutions are physically meaningful or are affected in any way by the size of our domain and the application of periodic conditions at the edges. To investigate this, we have repeated the calculations for $C a=10$ doubling the size of the computational domain and the long-time profile of the interfacial height is presented in Fig. 7. Note that the solution for the short domain have been extended periodically in the $x$-direction. Comparing the results for the two domains we find that, although the profiles present some similarities, they are far from identical. Nevertheless, we observe that in both cases the structures evolve by a rough doubling of the spatial periodicity that results from merging of neighbouring crest, with the growing hump "hesitating" between the respective electrode protrusions. Still, we are unable to conclude if the evolution by period-doubling is a general trend or is dictated by the imposed periodic boundary conditions. For $\mathrm{Ca}=20$ the solutions using the sort or long domain are identical and are not presented here for conciseness.

In practice in order to produce well-ordered polymeric microstructures over large distances it is important to operate in conditions where the induced structures are periodic. Apart from using the applied voltage to control the induced patterns, as shown above, it is also possible to tune the film thickness and the geometrical

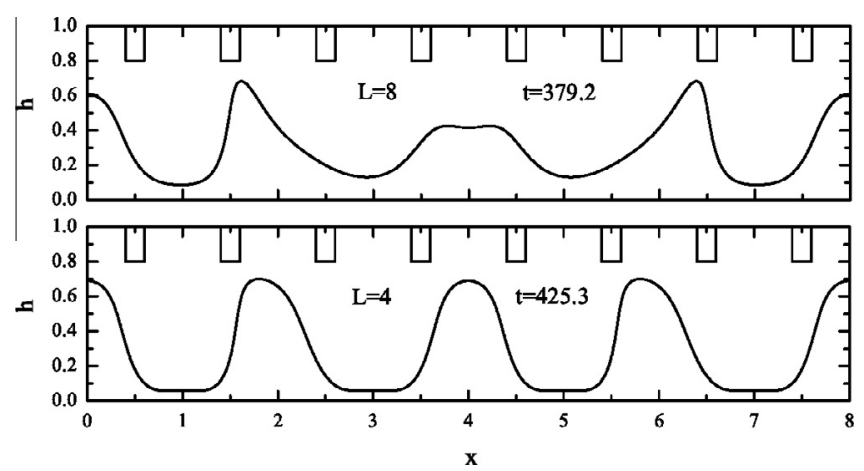

Fig. 7. Long time profiles of the liquid-air interface for different sizes of the computational domain for $W i=0, C a=10, \epsilon_{1}=2.5, \epsilon_{2}=1, d=0.3, s=0.8, p=0.2$ $w=0.2$. characteristics of the top electrode such as the periodicity of the protrusion as well as their width and depth. In Fig. 8 we depict a flow map to investigate the various flow patterns that may arise for various distances of the protrusions and different mean initial thickness of the polymer. Our calculations indicate the existence of two different regimes. The circles denote the regime where the induced structures are periodic whereas the triangles correspond to nonperiodic solutions. These are separated by a curve with filled circles which is used to indicate near critical conditions. To determine whether the pattern is periodic or not, we evaluate the standard deviation of the position of each peak from the mean value, $\bar{h}_{p}$, when the highest peak has reached $h=0.7$. If the standard deviation is lower than 0.001 then the structure is considered periodic whereas for higher values the structure is considered to be non-periodic. From Fig. 8 we deduce that increasing the initial film thickness, $d$, the minimum distance of the protrusions decreases significantly and therefore the fabrication limit of the induced patterns decreases as well. A similar configuration to ours was studied recently by $\mathrm{Li}$ et al. [31] using a diffuse interface method and it would be useful to compare their predictions for the period limit of the protrusions against our calculations using the sharp interface approach. For the fluid properties $(\sigma=0.038 \mathrm{~N} / \mathrm{m})$ and applied voltage $(70 \mathrm{~V})$ that were used by Li et al. [31] we deduce that $C a \approx 11.4$. For this set of parameters we get that the predicted critical distance between the electrode protrusions is approximately equal to $s=0.85$ which is in very good agreement with the predictions presented by these authors.

\subsection{Viscoelastic fluid}

Next we proceed with our investigation taking into account the effect of viscoelasticity of the polymeric film by studying the case of a PTT fluid. To start with, we present in Fig. 9 contour plots for the velocity field, pressure and electric potential at $t=28.44$ for $W i=2, C a=20, d=0.3$ and $s=0.8$; for clarity we present here only part of our computational domain $(1 \leqslant x \leqslant 3)$. As in the case of the Newtonian fluid for the given set of parameters we end up with a symmetric flow field around the protrusions and the induced structures are also symmetric. The velocity in the normal direction at the crest is higher and as a result the crest reaches the same height sooner for the case of the viscoelastic fluid than a Newtonian fluid with similar surface tension. Though this trend agrees with the findings of linear theory (which suggests that the growth rate increases with the elasticity of the material $[22,23]$ ),

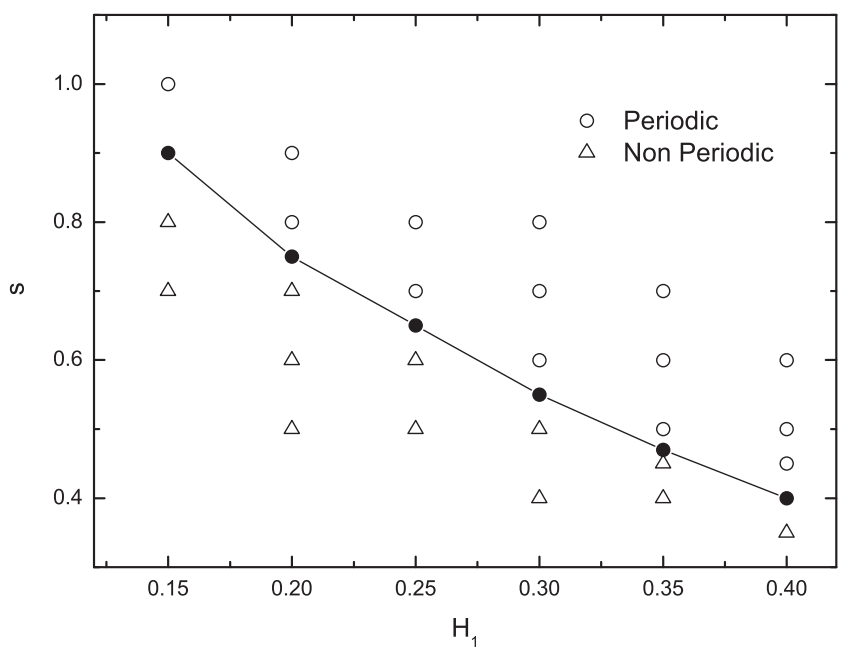

Fig. 8. Flow map for $W i=0, C a=20, \epsilon_{1}=2.5, \epsilon_{2}=1, p=0.2, w=0.2, L=4(s+w)$. 
(a)

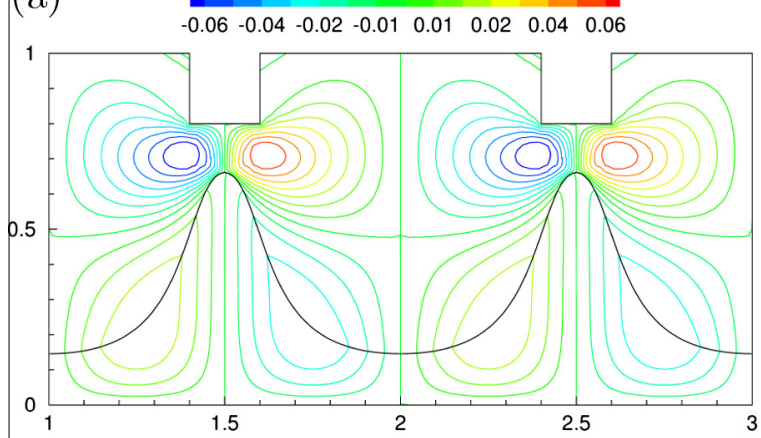

(c)

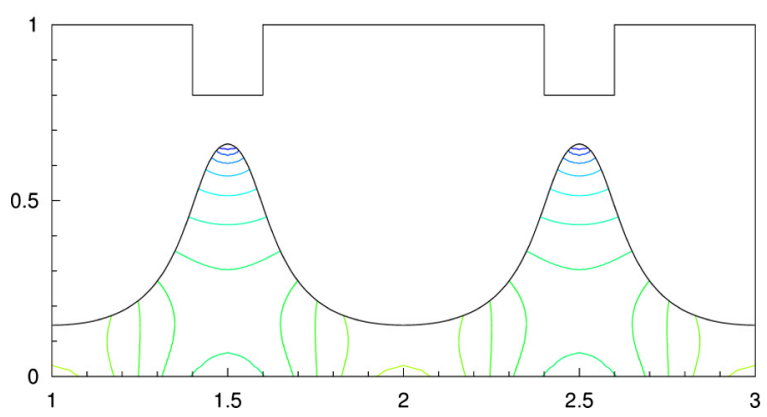

(b)

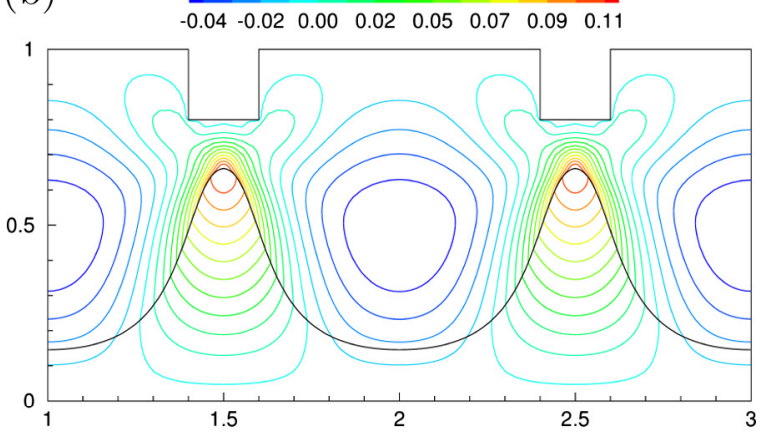

(d)

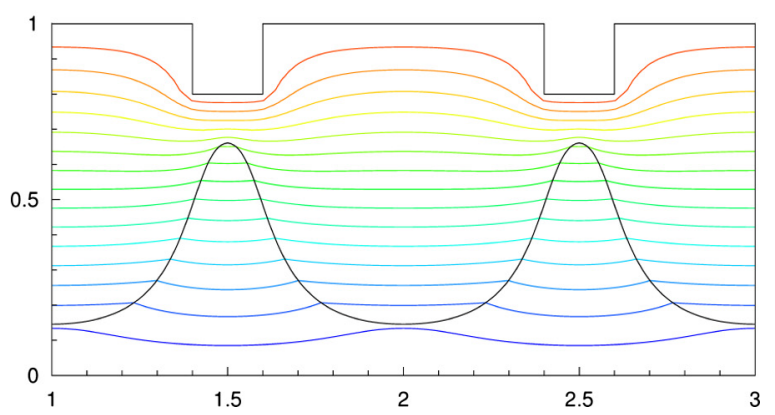

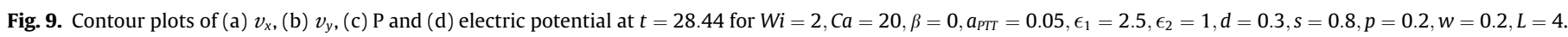
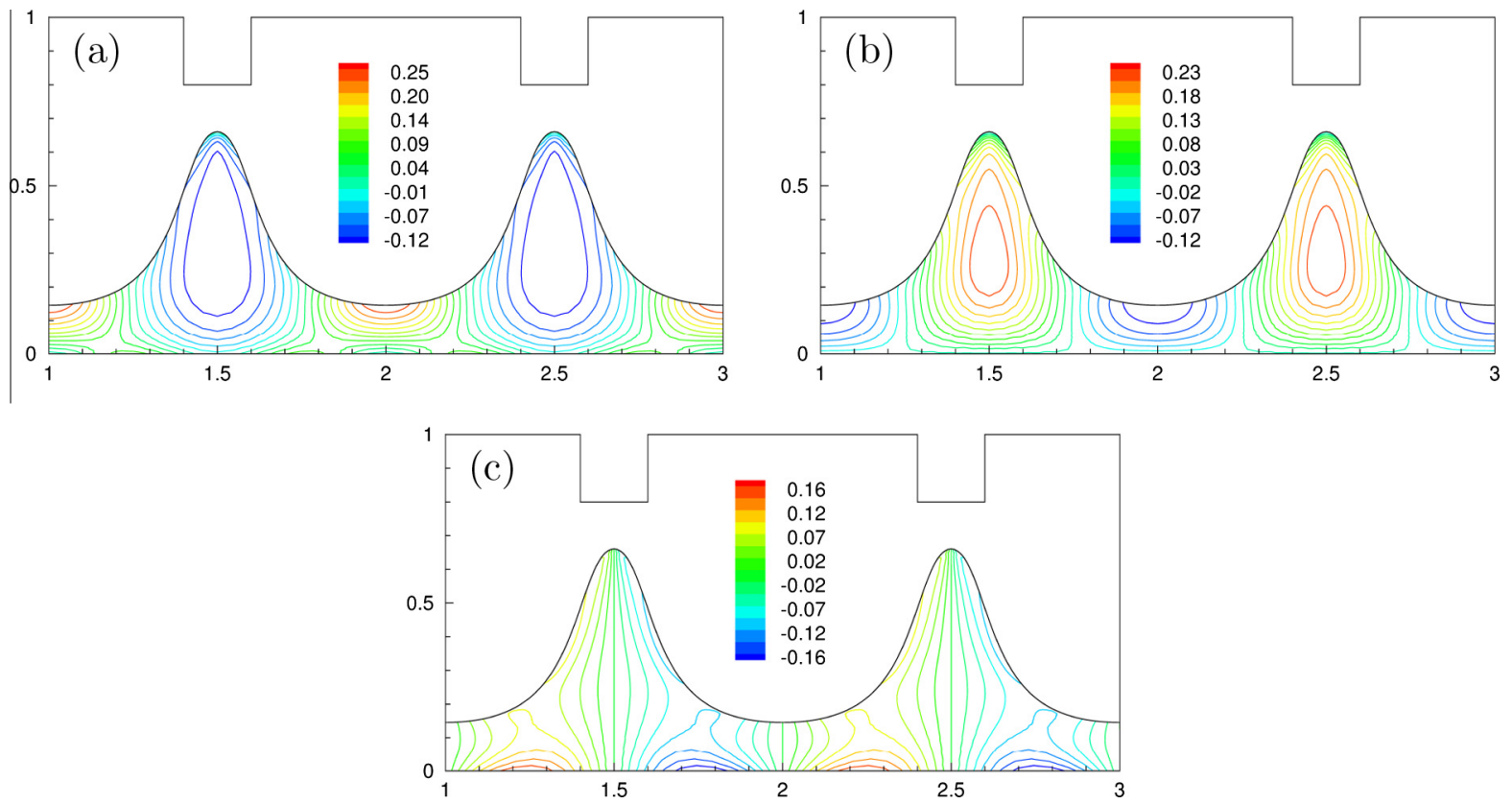

Fig. 10. Contour plots of (a) $\tau_{p, x x}$, (b) $\tau_{p, y y}$ and (c) $\tau_{p, x y}$ at $t=28.44$ for $W i=2, C a=20, \beta=0, a_{P T T}=0.05, \epsilon_{1}=2.5, \epsilon_{2}=1, d=0.3, s=0.8, p=0.2, w=0.2, L=4$.

the behaviour at finite deformations is presently interpreted differently in terms of the normal and shear stresses.

Iso-contours of the polymeric part of the stress components at an advanced stage of interfacial deformation is depicted in Fig. 10. The values of these components along the interface are plotted in Fig. 11 for two different times, corresponding to early (linear) and advanced (nonlinear) stage of the pillar growth. (We note that the contour lines given here and subsequently in this paper have been derived from the raw data without any post-processing for smoothing them. In spite of the sharp variations of stress components near the crests of the film, these contour lines remain smooth throughout the simulations. Apparently $\tau_{p, x x}$ and $\tau_{p, y y}$ develop boundary layers on the front, the accurate resolution of which required the local mesh refinement described earlier.)

As intuitively expected - and also concluded from Fig. 10b - the normal stress, $\tau_{p, y y}$, is extentional in the core of the crest and thus 

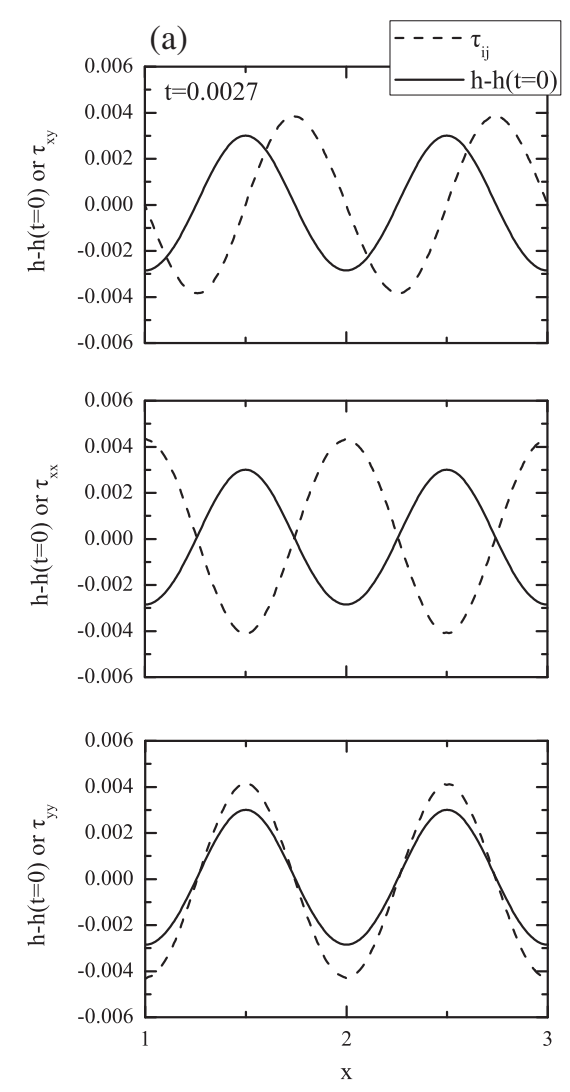
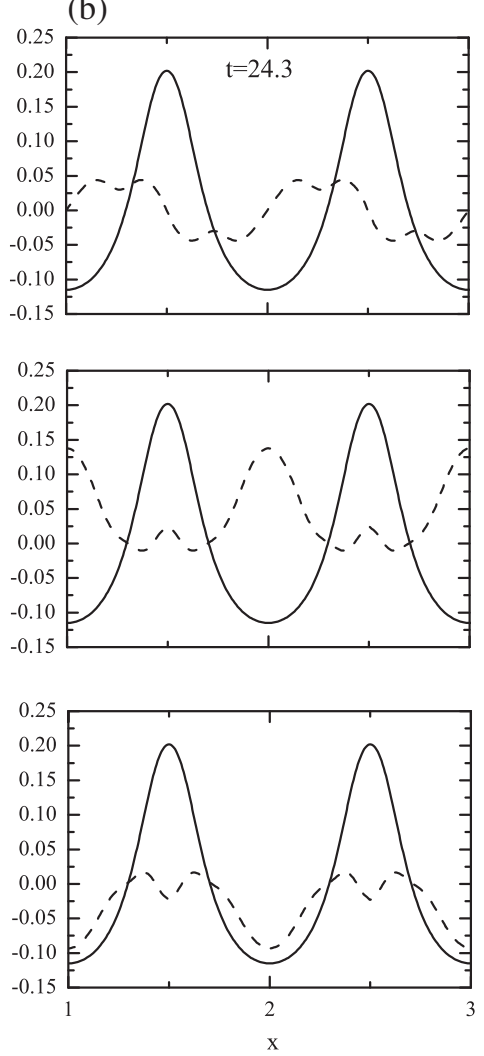

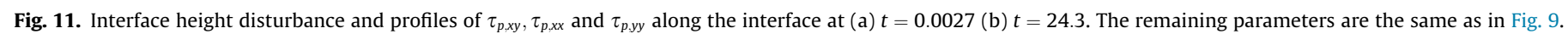

resists elongation of the pillar. However, Fig. 11 indicates that the relative intensity of this resistance decreases with deformation, as $\tau_{p, y y}$ is proportional to deformation at the linear stage but strongly displaced towards lower values later. The explanation of this relaxation is provided by the behaviour of the normal stress $\tau_{p, x x}$, which retains negative - i.e. compressive - values along the core of the pillar. The compressive $x$-component acts to thin the pilar and thus relaxes polymeric stresses in the $y$-direction. Finally, the shear component $\tau_{p, x y}$ also resists deformation according to Fig. 10c, and thus has a stabilizing role for highly deformed pillars.

It is noteworthy that according to $\mathrm{Wu}$ and Chou [22], who employ the lubrication approximation for analysis of the initial stages of deformation growth, the $x y$-component is the only one that survives in the linear limit and has a destabilizing effect. As it is shown in Fig. 11, this is actually true only at the very early stages of the flow. The magnitudes in Fig. 10c indicate that at finite growth normal stresses are dominant, being roughly two times larger than the $x y$ stress component. Thus, the usual assumption of reduced order models based on the lubrication approximation according to which the normal stresses are negligible - is clearly not valid at the late stages of the flow.

In Fig. 12a we examine the effect of the capillary number, $\mathrm{Ca}$, and present the evolution of the maximum amplitude of the interfacial perturbation for $W i=5$. Similarly to the case of a Newtonian fluid, for low $\mathrm{Ca}$ numbers we find that the amplitude initially grows and reaches a metastable state of a quasi-static low amplitude deformation, before eventually the pillars start growing again and reach the top electrode; as in the case of Newtonian fluids, the amplitude is found to increase with $\mathrm{Ca}$ (see Fig. 12c). We note that the power-law exponent of the dependence of the dimensionless amplitude $Z$ on $\epsilon \tau /\left(1-\tau^{2}\right)$ is not affected by the elasticity of the material. This indicates that the difference with the experimental value found in [9] should perhaps be attributed to the presence of free charge along the liquid-air interface which is not taken into account by our model. For higher values of the $\mathrm{Ca}$ number, the amplitude continuously grows until the liquid reaches the protrusions.

In Fig. 12b, we evaluate the threshold in the Ca number for the appearance of the metastable states for three different values of the Wi number in a similar manner to Fig. $4 \mathrm{~b}$. We find that the time spent in the metastable state is not affected by the elasticity of the material and this is also reflected in the value of the 'critical' $\mathrm{Ca}$ number which is $C a \approx 10$ independent of the value of the Wi number. On the other hand, we find that for high $\mathrm{Ca}$ the time that is needed to achieve $h_{\max }-h_{\min }=0.4$ decreases considerably with $W i$; This characteristic growth time is further reduced with increase of $\mathrm{Ca}$ as the role of surface tension becomes less significant. The above behaviour is probably due to the effect of shear and elongational thinning which is attributed to the PTT fluid by the finite value of the parameter $a_{P T T}$. When the capillary number is well beyond its critical value, the growth rate of the disturbances is large, resulting in high rates of deformation. High deformation rates render the effect of shear and elongational thinning - which facilitate growth of the pillars - gradually more significant.

The effect of the $\mathrm{Ca}$ number on the induced structures for the case of a viscoelastic fluid with $W i=1$ is presented in Fig. 13 where we plot the long-time profiles of the liquid-gas interface. Clearly, we find that decreasing the value of $\mathrm{Ca}$ and as the critical $\mathrm{Ca}$ for destabilization is approached the spatial periodicity of the structures is lost similarly to the case of a Newtonian fluid (see Fig. 6). Next, we keep the $C a$ number constant and vary the value of $W i$ (see Fig. 14). In the case of the Newtonian fluid the interface exhibits a periodic structure with pillars of equal size. With increasing $W i$ number a coarsening process takes place with the central pillars growing faster drawing fluid from their neighbours. It appears therefore that the bulk elasticity of the viscoelastic 

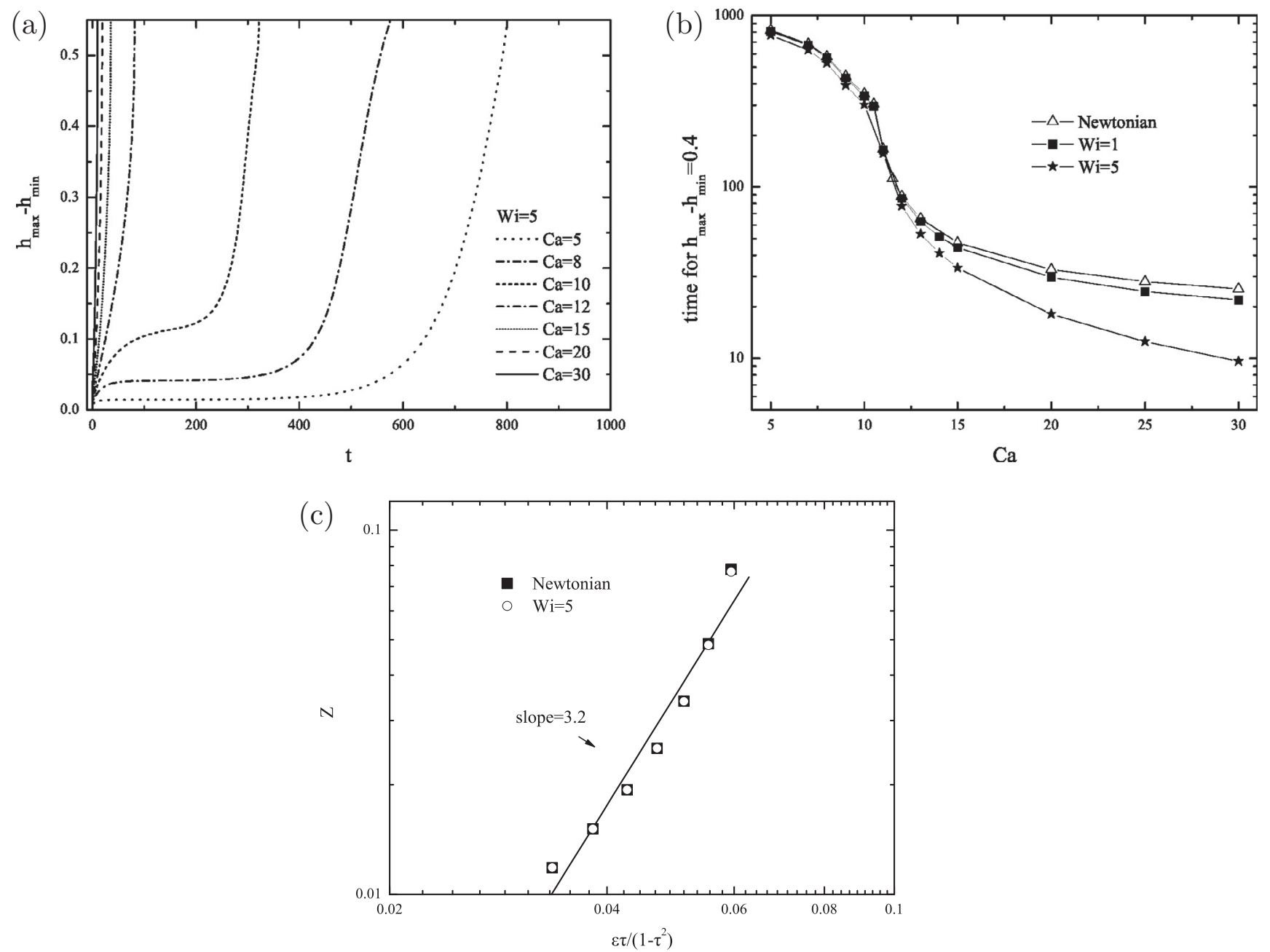

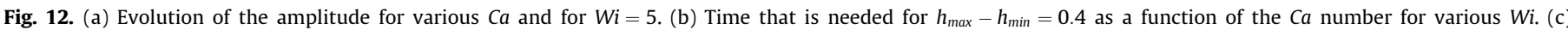

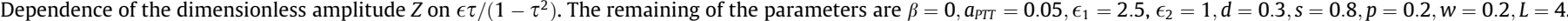
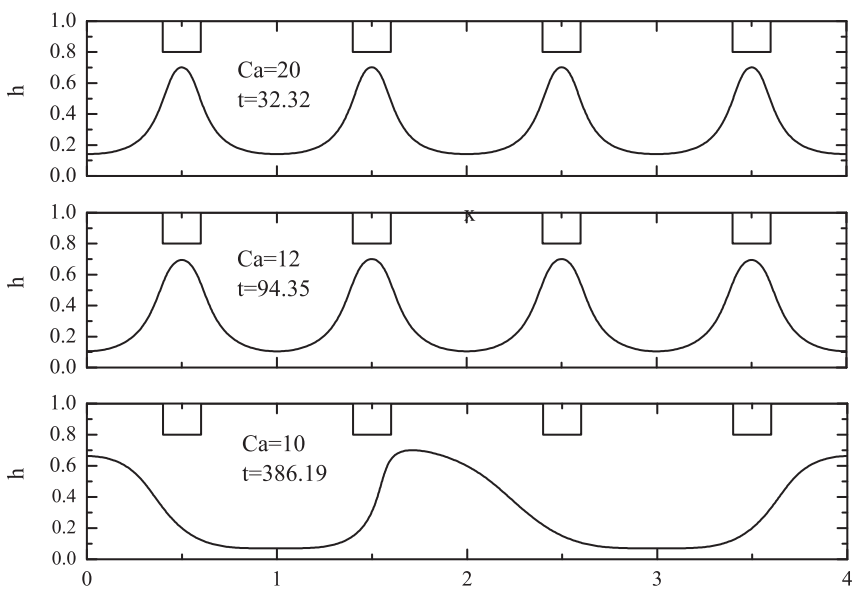

Fig. 13. Long time profiles of the liquid-air interface for various $\mathrm{Ca}$ and for $W i=1, \beta=0, a_{P T T}=0.05, \epsilon_{1}=2.5, \epsilon_{2}=1, d=0.3, s=0.8, p=0.2, w=0.2, L=4$.

material has a similar effect to surface tension, which can also be interpreted as the elasticity of the interface, and does not affect only the growth rate but also the wavelength of the disturbances. However it should be noted that the effect of the bulk elasticity appears to be much weaker that the effect of surface tension.

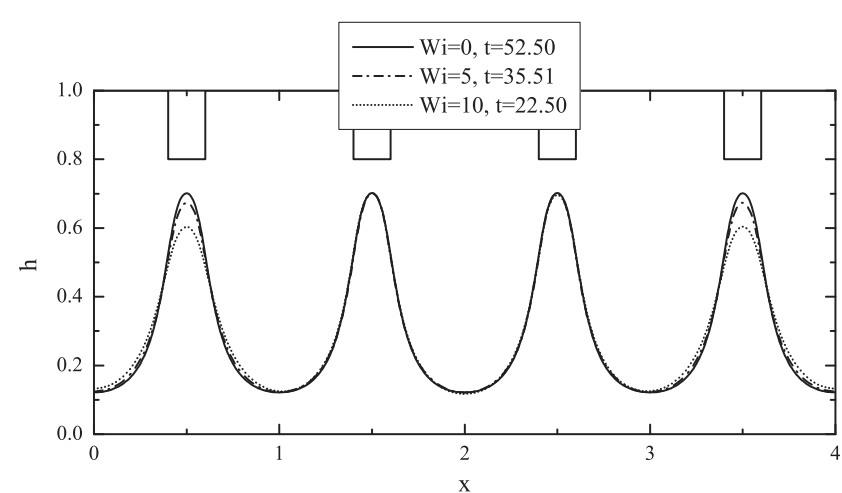

Fig. 14. Long time profiles of the liquid-air interface for various $W i$ and for $C a=15, \beta=0, a_{P T T}=0.05, \epsilon_{1}=2.5, \epsilon_{2}=1, d=0.3, s=0.8, p=0.2, w=0.2, L=4$.

Our simulations indicate that the effect of elasticity becomes larger for $\mathrm{Ca}$ numbers close to the 'critical' value whereas its effect decreases for high values of $\mathrm{Ca}$.

As was noticed above, in order to produce well-ordered polymeric micro-structures over large distances it is important to know under which conditions it is possible to get periodic structures. To examine the effect of elasticity on the fabrication limits of this process we produced the map shown in Fig. 15. Here each point in the 


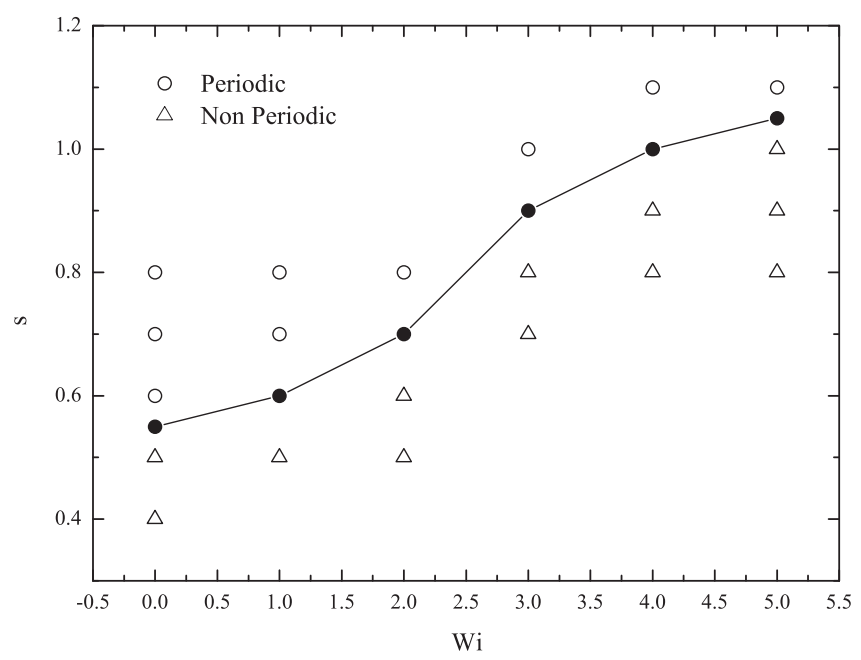

Fig. 15. Flow map for $\mathrm{Ca}=20, \beta=0, a_{P T T}=0.05, \epsilon_{1}=2.5, \epsilon_{2}=1, d=0.3, p=0.2$, $w=0.2, L=4(s+w)$

graph represents a simulation for the given value of the distance between the protrusions, $s$, and the corresponding Wi number. The open circles denote the case where we find solutions preserving the spatial periodicity whereas the triangles correspond to nonperiodic solutions. The solid circles on the other hand denote near critical conditions; we used the same criterion for critical conditions as described in Fig. 8. From this figure we deduce that the spacing between the protrusions, $s$, that results in periodic structures increases significantly with $W i$; this effect appears to saturate for high values of the Wi number. The critical $s$ will impose a fabrication limit on the width of the channels that can be manufactured, and it appears from the above that the more elastic the material, the higher the minimum width of the channels that can be achieved.

At this point, we should note that early studies using linear theory for homogeneous electric fields were suggesting that viscoelasticity affects the growth rate but leaves unaffected the wavelength of the most dangerous mode. We find this to be true also for heterogeneous electric fields at early times when the amplitude of the disturbances is small. However, as the amplitude becomes larger and non-linear effects come into play this is no longer true for the case of heterogeneous electric fields. Viscoelasticity does affect the wavelength of the induced structures and needs to be taken into account for designing the process efficiently.

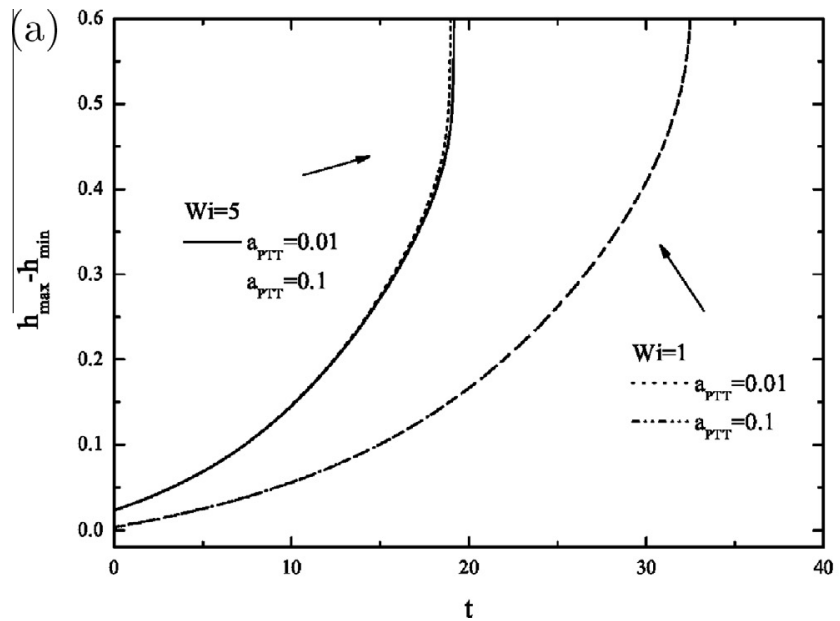

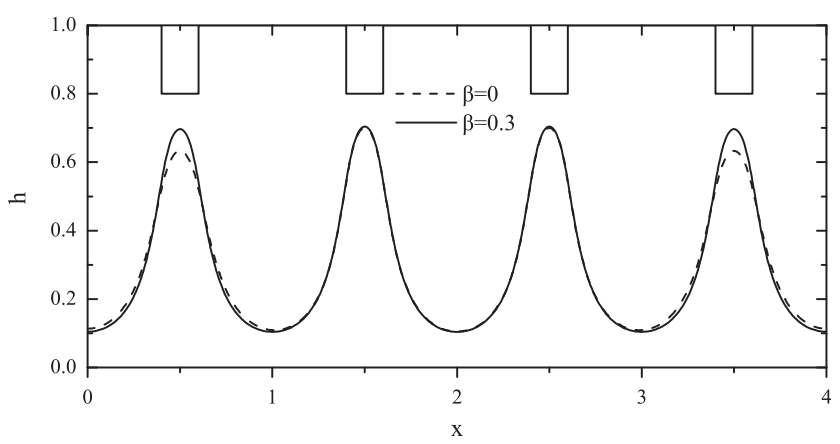

Fig. 17. Long time profiles of the liquid-air interface for two different values of $\beta$ and for $W i=5, C a=12, a_{P T T}=0.05, \quad \epsilon_{1}=2.5, \epsilon_{2}=1, d=0.3, s=0.8, p=0.2, w=0.2$, $L=4$.

\subsection{Effect of rheological parameters $a_{P T T}$ and $\beta$ and dielectric constant,} $\epsilon_{1}$

We continue our study by investigating the effect of the rheological parameters of the Phan-Thien Tanner model. Parameter $a_{P T T}$ controls the level of the elongational viscosity, and as it approaches zero the elongational viscosity increases to infinity. The influence of this parameter is more involved because it also affects the shear viscosity of the fluid. The predictions of the model for various values of this parameter can be found in [34]. To examine the effect of $a_{P T T}$, we have plotted in Fig. 16 the evolution of the amplitude of the maximum peak with time for two values of $W i$ and for various values of $a_{\text {PTT }}$. For $W i=1$ the curves are found to be identical for all three values of $a_{P T T}$, which suggests that shear and elongational thinning do not have a significant effect in this case. On the other hand, for $W i=5$ the dynamics are slightly affected with regards to the time that exponential growth is initiated. Nevertheless, the long-time profiles are not affected significantly and remain almost identical for all values of $a_{P T T}$; not shown here for conciseness. This is a clear indication that shear and elongational thinning may affect to some extent the dynamics of the flow but do not have any significant effect on the induced structures and therefore do not have to be taken into account in the design process.

The ratio of the solvent viscosity to the total viscosity, $\beta$, is also an important parameter because it also influences the level of viscoelasticity in the momentum balance. The effect of $\beta$ is examined in Figs. 16b and 17. As it is shown, the solvent viscosity has a significant impact on the flow dynamics and for low values of $\beta$ leads

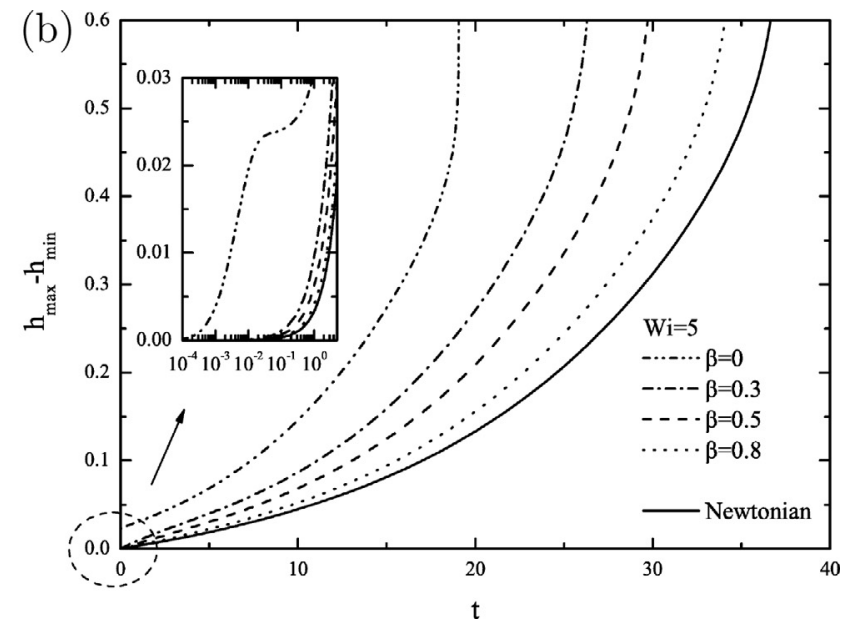

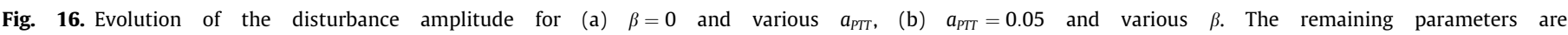
$C a=20, \epsilon_{1}=2.5, \epsilon_{2}=1, d=0.3, s=0.8, p=0.2, w=0.2, L=4$. 


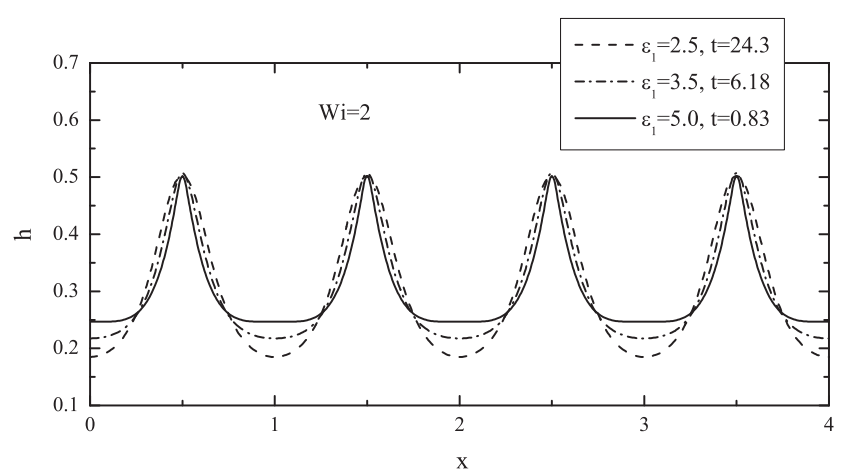

Fig. 18. Profiles of the liquid-air interface for three different values of $\epsilon_{1}$ and for $W i=2, \mathrm{Ca}=20, a_{P T T}=0.05, \beta=0, \epsilon_{2}=1, d=0.3, s=0.8, p=0.2, w=0.2, L=4$.

to an acceleration of the pillar growth. However, increasing further $\beta$, the Newtonian contribution increases considerably with respect to the polymeric one and the behaviour of the film approaches the Newtonian limit. The inset in Fig. 16b depicts the early time dynamics where it is clearly shown that the growth rate for small disturbances depends monotonically on $\beta$ in agreement with the results of linear theory presented in [23]. As it is shown in Fig. 17, for $\beta=0$ and for the particular choice of the remaining parameters, the film deviates from the Newtonian case. The addition of some Newtonian contribution reduces the levels of elasticity in the fluid decreasing in turn the fabrication limit on the period of the protrusions of the top electrode.

Finally, the effect of the dielectric constant, $\epsilon_{1}$, is depicted in Fig. 18 where we have plotted the liquid-interface profiles at the time instant for each case that the height of the pillars becomes equal to 0.5 . It is found that the pillars grow faster with increasing $\epsilon_{1}$ due to the higher intensity of the electric field that the liquid experiences. Regarding the shape of the formed pillars we find that with increasing $\epsilon_{1}$ the pillars become thinner at the peaks and flatter at the troughs; the pillars acquire a quasi triangular shape for the highest value of $\epsilon_{1}=5$.

\section{Conclusions}

We carried out a numerical investigation of the flow of both a Newtonian and a viscoelastic film under the action of an heterogeneous electric field imposed by the presence of a patterned electrode. The mixed finite element method was used combined with a quasi-elliptic mesh generation scheme, which allows an accurate description of the large deformations of the liquid-air interface. The viscoelastic behaviour of the polymeric film was modelled using the PTT constitutive law. Simulations up to high Weissenberg numbers were successful by using the EVSS-G/SUPG formulation for the numerical discretization and weighting of the constitutive equations. Attention was focused on the non-linear dynamics of the flow, and it was interrogated in particular how the dynamics is influenced by the rheological characteristics of the material and how in turn it affects the fabrication limits of this process.

We have shown that for low values of the $C a$ number a metastable state of finite amplitude interfacial deformation arises before eventually the perturbations grow until they reach the top electrode; during the late stages of the flow a coarsening process also takes place. Our non-linear simulations provide a better agreement with experimental observations for the amplitude of the pseudosteady state in comparison to the earlier predictions of linear theory [9]. We have shown that under the influence of a patterned electrode the effect of elasticity is more involved than what is suggested by linear theory for the case of an homogeneous electric field. We find that shear polymeric stresses are destabilizing at early times, as predicted by linear theory, but become stabilizing at later stages of the flow. Normal stresses, on the other hand, become increasingly important as the liquid-air interface deforms, destabilizing the film. It is shown that at late times normal stresses become dominant and cannot be ignored, as it is usually done under the lubrication approximation, for the accurate prediction of the flow dynamics. We also find that the fabrication limit on the period of the electrode protrusions appears to depend on the elasticity of the material, contrary to the predictions of linear theory of a constant most dangerous wavelength in the case of a flat electrode (homogeneous electric field). Finally, the amplitude of the pseudo-steady interfacial deformations appears to be unaffected by the elasticity of the material and the same is also true for the critical voltage below which these metastable states arise.

\section{Acknowledgement}

The authors would like to acknowledge the financial support by the General Secretariat of Research and Technology of Greece under the Action "Supporting Postdoctoral Researchers" (Grant No.: PE8/906), cofunded by the European Social Fund and National Resources.

\section{Appendix A. Finite element formulation}

The physical domain was discretized using triangular elements. We approximate the velocity vector, the position vector and the potential with six-node Lagrangian basis functions, $\psi^{j}$, and the pressure, the elastic stresses, as well as the velocity gradients with three-node Lagrangian basis functions, $\chi^{j}$.

For the momentum, mass balances and the Laplace equation for the potential, we employ the finite element/Galerkin method, which after applying the divergence theorem results in the following weak forms:

$$
\begin{aligned}
& \int_{V}\left[D_{i} \operatorname{Re}\left(\frac{\partial \mathbf{v}_{i}}{\partial t}+\mathbf{v}_{i} \cdot \nabla \mathbf{v}_{i}\right) \psi^{j}-P \nabla \psi^{j}+\nabla \psi^{j} \cdot \Sigma+2 \nabla \psi^{j} \cdot \gamma_{i}+S t \psi^{j} \mathbf{e}_{z}\right] d V \\
& -\int_{S}\left[\mathbf{n} \cdot T_{i}\right] \psi^{j} d S=0,
\end{aligned}
$$

$\int_{V}\left[\nabla \cdot \mathbf{v}_{i}\right] \chi^{j} d V=0$

$\int_{V}\left[\epsilon_{i} \nabla \phi_{i} \cdot \nabla \psi^{j}\right] d V-\int_{S}\left[\mathbf{n} \cdot \nabla \phi_{i}\right] \psi^{j} d S=0$.

where $d V$ and $d S$ are the differential volume and surface area, respectively. The surface integral that appears in the momentum equation is split into as many parts as the number of boundaries of the physical domain and the relevant boundary condition is applied therein.

The weak form of the mesh generation equations is derived similarly by applying the divergence theorem:

$$
\begin{aligned}
& \int_{V}[\alpha \cdot \nabla \eta] \cdot \nabla \psi^{j} d V=0, \\
& \int_{V}\left[\left(\delta \sqrt{\frac{x_{\xi}^{2}+y_{\xi}^{2}}{x_{\eta}^{2}+y_{\eta}^{2}}}+(1-\delta)\right) \nabla \xi\right] \cdot \nabla \psi^{j} d V=0 .
\end{aligned}
$$

The continuous approximation for the components of the velocity gradient tensor is written as

$\int_{V}\left[G-\nabla \mathbf{v}_{1}\right] \chi^{j} d V=0$.

The constitutive equation due to its hyperbolic character is discretized using the streamline upwind Petrov-Galerkin (SUPG) method proposed by [47] 
$\int_{V}\left[Y\left(\tau_{p, 1}\right) \Sigma+W i \hat{\Sigma}+2 W i(1-\beta) M_{1} \hat{\Pi}-2(1-\beta)\left(1-Y\left(\tau_{p, 1}\right)\right) M_{1} \Pi\right]$ $\omega^{j} d V=0$

where $\Pi=1 / 2\left(G+G^{T}\right)$ and the definition of the Gordon-Schowalter derivative is given by

$\widehat{X}=\frac{\partial X}{\partial t}+\mathbf{v}_{1} \cdot \nabla X-\left(\nabla \mathbf{v}_{1}-\xi_{s} \Pi\right)^{T} \cdot X-X \cdot\left(\nabla \mathbf{v}_{1}-\xi_{s} \Pi\right)$.

The weighting function $\omega^{j}$ is formed from the finite element basis function for the elastic stress components according to

$\omega^{j}=\chi^{j}+\frac{h}{\left|\mathbf{v}_{1}\right|} \nabla \chi^{j}$

where $\left|\mathbf{v}_{1}\right|$ is the mean velocity and $h$ is a characteristic length in each element. The mean velocity $\left|\mathbf{v}_{1}\right|$ in an element is defined as the mean value of the velocity at the vertices of the corresponding element. As a characteristic length, $h$, we used the square root of the area of each triangular element.

\section{References}

[1] S.Y. Chou, L. Zhuang, Lithographically induced self-assembly of periodic polymer micropillar arrays, J. Vac. Sci. Technol. B: Microelectron. Nanometer Struct. 17 (6) (1999) 31973202

[2] E. Schäffer, T. Thurn-Albrecht, T.P. Russell, U. Steiner, Electrically induced structure formation and pattern transfer, Nature 403 (6772) (2000) 874-877.

[3] E. Schäffer, T. Thurn-Albrecht, T.P. Russell, U. Steiner, Electrohydrodynamic instabilities in polymer films, Europhys. Lett. (EPL) 53 (4) (2001) 518-524.

[4] Z. Lin, T. Kerle, S.M. Baker, D.A. Hoagland, E. Schäffer, U. Steiner, T.P. Russell, Electric field induced instabilities at liquid/liquid interfaces, J. Chem. Phys. 114 (5) (2001) 2377

[5] Z. Lin, T. Kerle, T.P. Russell, E. Schäffer, U. Steiner, Structure formation at the interface of liquid/liquid bilayer in electric field, Macromolecules 35 (10) (2002) 3971-3976.

[6] M.D. Morariu, N.E. Voicu, E. Schäffer, Z. Lin, T.P. Russell, U. Steiner, Hierarchical structure formation and pattern replication induced by an electric field, Nature Mater. 2 (1) (2003) 48-52.

[7] L.F. Pease, W.B. Russel, Linear stability analysis of thin leaky dielectric films subjected to electric fields, J. NonNewton. Fluid Mech. 102 (2) (2002) 233-250.

[8] V. Shankar, A. Sharma, Instability of the interface between thin fluid films subjected to electric fields, J. Colloid Interface Sci. 274 (2004) 294-308.

[9] J. Heier, J. Groenewold, U. Steiner, Pattern formation in thin polymer films by spatially modulated electric fields, Soft Matter 5 (2009) 3997.

[10] R.V. Craster, O.K. Matar, Electrically induced pattern formation in thin leaky dielectric films, Phys. Fluids 17 (2005) 032104.

[11] D. Merkt, A. Pototsky, M. Bestehorn, U. Thiele, Long-wave theory of bounded two-layer films with a free liquid-liquid interface: short- and long-time evolution, Phys. Fluids 17 (2005) 064104

[12] R. Verma, A. Sharma, K. Kargupta, J. Bhaumik, Electric field induced instability and pattern formation in thin liquid films, Langmuir 21 (8) (2005) 3710-3721.

[13] N. Wu, L.F. Pease, W.B. Russel, Electric-field-induced patterns in thin polymer films:? Weakly nonlinear and fully nonlinear evolution, Langmuir 21 (26) (2005) 12290-12302.

[14] N. Wu, W.B. Russel, Dynamics of the formation of polymeric microstructures induced by electrohydrodynamic instability, Appl. Phys. Lett. 86 (24) (2005) 241912.

[15] D. Bandyopadhyay, A. Sharma, Electric field induced instabilities in thin confined bilayers, J. Colloid Interface Sci. 311 (2) (2007) 595-608.

[16] D. Bandyopadhyay, A. Sharma, U. Thiele, P.D.S. Reddy, Electric-field-induced interfacial instabilities and morphologies of thin viscous and elastic bilayers Langmuir 25 (16) (2009) 91089118.

[17] P.D.S. Reddy, D. Bandyopadhyay, A. Sharma, Self-organized ordered arrays of core-shell columns in viscous bilayers formed by spatially varying electric fields, J. Phys. Chem. C 114 (49) (2010) 21020-21028.

[18] S.A. Roberts, S. Kumar, Electrohydrodynamic instabilities in thin liquid trilayer films, Phys. Fluids 22 (12) (2010) 122102.

[19] P.D.S. Reddy, D. Bandyopadhyay, A. Sharma, Electric-field-induced instabilities in thin liquid trilayers confined between patterned electrodes, J. Phys. Chem. C 116 (43) (2012) 2284722858
[20] S.A. Roberts, S. Kumar, AC electrohydrodynamic instabilities in thin liquid films, J. Fluid Mech. 631 (2009) 255.

[21] P. Gambhire, R.M. Thaokar, Linear stability analysis of electrohydrodynamic instabilities at fluid interfaces in the small feature limit, Euro. Phys. J. E 34 (8) (2011) 1-12.

[22] L. Wu, S. Chou, Electrohydrodynamic instability of a thin film of viscoelastic polymer underneath a lithographically manufactured mask, J. Nonnewton. Fluid Mech. 125 (2-3) (2005) 91-99.

[23] G. Tomar, V. Shankar, A. Sharma, G. Biswas, Electrohydrodynamic instability of a confined viscoelastic liquid film, J. Nonnewton. Fluid Mech. 143 (2-3) (2007) $120-130$.

[24] L. Espín, A. Corbett, S. Kumar, Electrohydrodynamic instabilities in thin viscoelastic films AC and DC fields, J. Nonnewton. Fluid Mech. 196 (2013) 102111.

[25] D. Bandyopadhyay, P. Dinesh Sankar Reddy, A. Sharma, Electric field and van der Waals force induced instabilities in thin viscoelastic bilayers, Phys. Fluids 24 (7) (2012) 074106-1-074106-29.

[26] L.F. Pease, W.B. Russel, Electrostatically induced submicron patterning of thin perfect and leaky dielectric films: a generalized linear stability analysis, J. Chem. Phys. 118 (8) (2003) 3790-3803.

[27] L.F. Pease, W.B. Russel, Limitations on length scales for electrostatically induced submicrometer pillars and holes, Langmuir 20 (3) (2004) 795-804.

[28] D. Kim, W. Lu, Interface instability and nanostructure patterning, Comput. Mater. Sci. 38 (2) (2006) 418-425.

[29] H.K. Yeoh, Q. Xu, O.A. Basaran, Equilibrium shapes and stability of a liquid film subjected to a nonuniform electric field, Phys. Fluids 19 (11) (2007) 114111.

[30] Q. Yang, B.Q. Li, Y. Ding, A numerical study of nanoscale electrohydrodynamic patterning in a liquid film, Soft Matter 9 (2013) 3412.

[31] H. Li, W. Yu, L. Zhang, Z. Liu, K.E. Brown, E. Abraham, S. Cargill, C. Tonry, M.K. Patel, C. Bailey, M.P. Desmulliez, Simulation and modelling of sub-30 nm polymeric channels fabricated by electrostatic induced lithography, RSC Adv. 3 (2013) 11839.

[32] N. Phan-Thien, R.I. Tanner, A new constitutive equation derived from network theory, J. NonNewton. Fluid Mech. 2 (4) (1977) 353-365.

[33] N. Phan-Thien, A nonlinear network viscoelastic model, J. Rheol. 22 (3) (1978) 259-283.

[34] G. Karapetsas, J. Tsamopoulos, On the stick-slip flow from slit and cylindrical dies of a Phan-Thien and Tanner fluid model. II. Linear stability analysis, Phys. Fluids 25 (9) (2013) 093105.

[35] D. Rajagopalan, R.C. Armstrong, R.A. Brown, Finite element methods for calculation of steady, viscoelastic flow using constitutive equations with a newtonian viscosity, J. NonNewton. Fluid Mech. 36 (1990) 159192.

[36] R.A. Brown, M.J. Szady, P.J. Northey, R.C. Armstrong, On the numerical stability of mixed finite-element methods for viscoelastic flows governed by differential constitutive equations, Theoret. Comput. Fluid Dyn. 5 (2) (1993) 77106.

[37] G. Karapetsas, J. Tsamopoulos, Steady extrusion of viscoelastic materials from an annular die, J. Non Newt. Fluid Mech. 154 (2008) 136152.

[38] G. Karapetsas, J. Tsamopoulos, On the stick-slip flow from slit and cylindrical dies of a Phan-Thien and tanner fluid model. i. steady state, Phys. Fluids 21 (12) (2009) 123101.

[39] G. Karapetsas, J. Tsamopoulos, Transient squeeze flow of viscoplastic materials, J. Non Newt. Fluid Mech. 133 (2006) 35-56.

[40] J. Papaioannou, G. Karapetsas, Y. Dimakopoulos, J. Tsamopoulos, Injection of a viscoplastic material inside a tube or between two parallel disks: conditions for wall detachment of the advancing front, J. Rheol. 53 (5) (2009) 1155-1191.

[41] G. Karapetsas, O.K. Matar, P. Valluri, K. Sefiane, Convective rolls and hydrothermal waves in evaporating sessile drops, Langmuir 28 (31) (2012) $11433-11439$.

[42] K. Tsiveriotis, R.A. Brown, Boundary-conforming mapping applied to computations of highly deformed solidification interfaces, Int. J. Numer. Meth. Fluids 14 (8) (1992) 9811003.

[43] K. Christodoulou, L. Scriven, Discretization of free surface flows and other moving boundary problems, J. Comput. Phys. 99 (1) (1992) 39-55.

[44] Y. Dimakopoulos, J. Tsamopoulos, A quasi-elliptic transformation for moving boundary problems with large anisotropic deformations, J. Comput. Phys. 192 (2) (2003) 494522

[45] N. Chatzidai, A. Giannousakis, Y. Dimakopoulos, J. Tsamopoulos, On the elliptic mesh generation in domains containing multiple inclusions and undergoing large deformations, J. Comput. Phys. 228 (6) (2009) 1980-2011.

[46] N. Wu, M.E. Kavousanakis, W.B. Russel, Coarsening in the electrohydrodynamic patterning of thin polymer films, Phys. Rev. E 81 (2010) 020306

[47] A.N. Brooks, T.J. Hughes, Streamline upwind/Petrov-Galerkin formulations for convection dominated flows with particular emphasis on the incompressible Navier-stokes equations, Comput. Meth. Appl. Mech. Eng. 32 (13) (1982) 199_ 259. 